\title{
Shrinking Projection Method of Common Fixed Point Problems for Discrete Asymptotically Strictly Pseudocontractive Semigroups and Mixed Equilibrium Problems in Hilbert Spaces
}

\author{
Pattanapong Tianchai \\ Faculty of Science, Maejo University, Chiangmai 50290, Thailand \\ Correspondence should be addressed to Pattanapong Tianchai, pattana@mju.ac.th \\ Received 13 July 2011; Accepted 18 August 2011 \\ Academic Editor: A. Levy
}

Copyright (C) 2011 Pattanapong Tianchai. This is an open access article distributed under the Creative Commons Attribution License, which permits unrestricted use, distribution, and reproduction in any medium, provided the original work is properly cited.

This paper is concerned with a common element of the set of common fixed point for a discrete asymptotically strictly pseudocontractive semigroup and the set of solutions of the mixed equilibrium problems in Hilbert spaces. The strong convergence theorem for the above two sets is obtained by a general iterative scheme based on the shrinking projection method which extends and improves the corresponding ones due to Kim [Proceedings of the Asian Conference on Nonlinear Analysis and Optimization (Matsue, Japan, 2008), 139-162].

\section{Introduction}

Throughout this paper, we always assume that $C$ is a nonempty closed convex subset of a real Hilbert space $H$ with inner product and norm denoted by $\langle\cdot, \cdot\rangle$ and $\|\cdot\|$, respectively. The domain of the function $\varphi: C \rightarrow \mathbb{R} \cup\{+\infty\}$ is the set

$$
\operatorname{dom} \varphi=\{x \in C: \varphi(x)<+\infty\}
$$

Let $\varphi: C \rightarrow \mathbb{R} \cup\{+\infty\}$ be a proper extended real-valued function, and let $\Phi$ be a bifunction from $C \times C$ into $\mathbb{R}$ such that $C \cap \operatorname{dom} \varphi \neq \emptyset$, where $\mathbb{R}$ is the set of real numbers. The so-called mixed equilibrium problem is to find $x \in C$ such that

$$
\Phi(x, y)+\varphi(y)-\varphi(x) \geq 0, \quad \forall y \in C
$$


The set of solutions of problem (1.2) is denoted by $\operatorname{MEP}(\Phi, \varphi)$; that is,

$$
\operatorname{MEP}(\Phi, \varphi)=\{x \in C: \Phi(x, y)+\varphi(y)-\varphi(x) \geq, \forall y \in C\}
$$

It is obvious that if $x$ is a solution of problem (1.2) then $x \in \operatorname{dom} \varphi$. As special cases of problem (1.2), we have the following.

(i) If $\varphi=0$, then problem (1.2) is reduced to find $x \in C$ such that

$$
\Phi(x, y) \geq 0, \quad \forall y \in C
$$

We denote by $E P(\Phi)$ the set of solutions of equilibrium problem, problem (1.4) which was studied by Blum and Oettli [1].

(ii) If $\Phi(x, y)=\langle B x, y-x\rangle$ for all $x, y \in C$ where a mapping $B: C \rightarrow H$, then problem (1.4) is reduced to find $x \in C$ such that

$$
\langle B x, y-x\rangle \geq 0, \quad \forall y \in C
$$

We denote by $\operatorname{VI}(C, B)$ the set of solutions of variational inequality problem, problem (1.5) which was studied by Hartman and Stampacchia [2].

(iii) If $\Phi=0$, then problem (1.2) is reduced to find $x \in C$ such that

$$
\varphi(y)-\varphi(x) \geq 0, \quad \forall y \in C
$$

We denote by $\operatorname{Argmin}(\varphi)$ the set of solutions of minimize problem.

Recall that $P_{C}$ is the metric projection of $H$ onto $C$; that is, for each $x \in H$, there exists the unique point in $P_{C} x \in C$ such that $\left\|x-P_{C} x\right\|=\min _{y \in C}\|x-y\|$. A mapping $T: C \rightarrow C$ is called nonexpansive if $\|T x-T y\| \leq\|x-y\|$ for all $x, y \in C$, and a mapping $f: C \rightarrow C$ is called a contraction if there exists a constant $\alpha \in(0,1)$ such that $\|f(x)-f(y)\| \leq \alpha\|x-y\|$ for all $x, y \in C$. A point $x \in C$ is a fixed point of $T$ provided $T x=x$. We denote by $F(T)$ the set of fixed points of $T$; that is, $F(T)=\{x \in C: T x=x\}$. If $C$ is a nonempty bounded closed convex subset of $H$ and $T$ is a nonexpansive mapping of $C$ into itself, then $F(T)$ is nonempty (see [3]).

Iterative methods are often used to solve the fixed point equation $T x=x$. The most well-known method is perhaps the Picard successive iteration method when $T$ is a contraction. Picard's method generates a sequence $\left\{x_{n}\right\}$ successively as $x_{n+1}=T x_{n}$ for all $n \in \mathbb{N}$ with $x_{1}=x$ chosen arbitrarily, and this sequence converges in norm to the unique fixed point of $T$. However, if $T$ is not a contraction (for instance, if $T$ is a nonexpansive), then Picard's successive iteration fails, in general, to converge. Instead, Mann's iteration method for a nonexpansive mapping $T$ (see [4]) prevails and generates a sequence $\left\{x_{n}\right\}$ recursively by

$$
x_{n+1}=\alpha_{n} x_{n}+\left(1-\alpha_{n}\right) T x_{n}, \quad \forall n \in \mathbb{N},
$$

where $x_{1}=x \in C$ chosen arbitrarily and the sequence $\left\{\alpha_{n}\right\}$ lies in the interval $[0,1]$. Recall that a mapping $T: C \rightarrow C$ is said to be as follows. 
(i) $\mathcal{\kappa}$-strictly pseudocontractive (see [5]) if there exists a constant $\kappa \in[0,1)$ such that

$$
\|T x-T y\|^{2} \leq\|x-y\|^{2}+\kappa\|(I-T) x-(I-T) y\|^{2}, \quad \forall x, y \in C,
$$

in brief, we use $\kappa$-SPC to denote the $\kappa$-strictly pseudocontractive, it is obvious that $T$ is a nonexpansive if and only if $T$ is a 0-SPC.

(ii) Asymptotically $\kappa$-SPC (see [6]) if there exists a constant $\kappa \in[0,1)$ and a sequence $\left\{\gamma_{n}\right\}$ of nonnegative real numbers with $\lim _{n \rightarrow \infty} \gamma_{n}=0$ such that

$$
\left\|T^{n} x-T^{n} y\right\|^{2} \leq\left(1+\gamma_{n}\right)\|x-y\|^{2}+\kappa\left\|\left(I-T^{n}\right) x-\left(I-T^{n}\right) y\right\|^{2}, \quad \forall x, y \in C,
$$

for all $n \in \mathbb{N}$, if $\kappa=0$ then $T$ is an asymptotically nonexpansive with $k_{n}=\sqrt{1+\gamma_{n}}$ for all $n \in \mathbb{N}$; that is, $T$ is an asymptotically nonexpansive (see [7]) if there exists a sequence $\left\{k_{n}\right\} \subset[1, \infty)$ with $\lim _{n \rightarrow \infty} k_{n}=1$ such that

$$
\left\|T^{n} x-T^{n} y\right\| \leq k_{n}\|x-y\|, \quad \forall x, y \in C,
$$

for all $n \in \mathbb{N}$, it is known that the class of $\kappa$-SPC mappings, and the classes of asymptotically $\kappa$-SPC mappings are independent (see [8]).

The Mann's algorithm for nonexpansive mappings has been extensively investigated (see $[5,9,10]$ and the references therein). One of the well-known results is proven by Reich [10] for a nonexpansive mapping $T$ on $C$, which asserts the weak convergence of the sequence $\left\{x_{n}\right\}$ generated by (1.7) in a uniformly convex Banach space with a Frechet differentiable norm under the control condition $\sum_{n=1}^{\infty} \alpha_{n}\left(1-\alpha_{n}\right)=\infty$. Recently, Marino and $\mathrm{Xu}$ [11] devoloped and extended Reich's result to SPC mapping in Hilbert space setting. More precisely, they proved the weak convergence of the Mann's iteration process (1.7) for a $\kappa$-SPC mapping $T$ on $C$, and, subsequently, this result was improved and carried over the class of asymptotically $\kappa$-SPC mappings by Kim and $\mathrm{Xu}[12]$.

It is known that the Mann's iteration (1.7) is in general not strongly convergent (see [13]). The strong convergence is guaranteed and has been proposed by Nakajo and Takahashi [14], they modified the Mann's iteration method (1.7) which is to find a fixed point of a nonexpansive mapping by a hybrid method, which called the shrinking projection method (or the CQ method) as the following theorem.

Theorem NT. Let $C$ be a nonempty closed convex subset of a real Hilbert space $H$. Let $T$ be a nonexpansive mapping of $C$ into itself such that $F(T) \neq \emptyset$. Suppose that $x_{1}=x \in C$ chosen arbitrarily, and let $\left\{x_{n}\right\}$ be the sequence defined by

$$
\begin{gathered}
y_{n}=\alpha_{n} x_{n}+\left(1-\alpha_{n}\right) T x_{n}, \\
C_{n}=\left\{z \in C:\left\|y_{n}-z\right\| \leq\left\|x_{n}-z\right\|\right\}, \\
Q_{n}=\left\{z \in C:\left\langle x_{n}-z, x_{1}-x_{n}\right\rangle \geq 0\right\}, \\
x_{n+1}=P_{C_{n} \cap Q_{n}}\left(x_{1}\right), \quad \forall n \in \mathbb{N},
\end{gathered}
$$

where $0 \leq \alpha_{n} \leq \alpha<1$. Then $\left\{x_{n}\right\}$ converges strongly to $P_{F(T)}\left(x_{1}\right)$. 
Subsequently, Marino and Xu [15] introduced an iterative scheme for finding a fixed point of a $\kappa$-SPC mapping as the following theorem.

Theorem MX. Let $C$ be a closed convex subset of a Hilbert space $H$, and, $T: C \rightarrow C$ be a $\kappa$-SPC mapping for some $0 \leq \kappa<1$. Assume that $F(T) \neq \emptyset$. Suppose that $x_{1}=x \in C$ chosen arbitrarily, and let $\left\{x_{n}\right\}$ be the sequence defined by

$$
\begin{gathered}
y_{n}=\alpha_{n} x_{n}+\left(1-\alpha_{n}\right) T x_{n}, \\
C_{n}=\left\{z \in C:\left\|y_{n}-z\right\|^{2} \leq\left\|x_{n}-z\right\|^{2}+\left(1-\alpha_{n}\right)\left(\kappa-\alpha_{n}\right)\left\|x_{n}-T x_{n}\right\|^{2}\right\}, \\
Q_{n}=\left\{z \in C:\left\langle x_{n}-z, x_{1}-x_{n}\right\rangle \geq 0\right\}, \\
x_{n+1}=P_{C_{n} \cap Q_{n}}\left(x_{1}\right), \quad \forall n \in \mathbb{N},
\end{gathered}
$$

where $0 \leq \alpha_{n}<1$. Then the sequence $\left\{x_{n}\right\}$ converges strongly to $P_{F(T)}\left(x_{1}\right)$.

Quite recently, Kim and Xu [12] has improved and carried Theorem MX over the more wider class of asymptotically $\kappa$-SPC mappings as the following theorem.

Theorem KX. Let $C$ be a closed convex subset of a Hilbert space $H$, and, $T: C \rightarrow C$ be an asymptotically $\kappa$-SPC mapping for some $0 \leq \kappa<1$ and a bounded sequence $\left\{\gamma_{n}\right\} \subset[0, \infty)$ such that $\lim _{n \rightarrow \infty} \gamma_{n}=0$. Assume that $F(T)$ is a nonempty bounded subset of $C$. Suppose that $x_{1}=x \in C$ chosen arbitrarily, and let $\left\{x_{n}\right\}$ be the sequence defined by

$$
\begin{gathered}
y_{n}=\alpha_{n} x_{n}+\left(1-\alpha_{n}\right) T^{n} x_{n}, \\
C_{n}=\left\{z \in C:\left\|y_{n}-z\right\|^{2} \leq\left\|x_{n}-z\right\|^{2}+\left(\kappa-\alpha_{n}\left(1-\alpha_{n}\right)\right)\left\|x_{n}-T^{n} x_{n}\right\|^{2}+\theta_{n}\right\}, \\
Q_{n}=\left\{z \in C:\left\langle x_{n}-z, x_{1}-x_{n}\right\rangle \geq 0\right\}, \\
x_{n+1}=P_{C_{n} \cap Q_{n}}\left(x_{1}\right), \quad \forall n \in \mathbb{N},
\end{gathered}
$$

where $\theta_{n}=\Delta_{n}^{2}\left(1-\alpha_{n}\right) \gamma_{n} \rightarrow 0$ as $n \rightarrow \infty, \Delta_{n}=\sup \left\{\left\|x_{n}-z\right\|: z \in F(T)\right\}<\infty$, and $0 \leq \alpha_{n}<1$ such that $\limsup _{n \rightarrow \infty} \alpha_{n}<1-\kappa$. Then the sequence $\left\{x_{n}\right\}$ converges strongly to $P_{F(T)}\left(x_{1}\right)$.

Recall that a discrete family $\mathcal{S}=\left\{T_{n}: n \geq 0\right\}$ of self-mappings of $C$ is said to be a Lipschitzian semigroup on $C$ if the following conditions are satisfied.

(1) $T_{0}=I$ where $I$ denotes the identity operator on $C$.

(2) $T_{n+m} x=T_{n} T_{m} x, \forall n, m \geq 0, \forall x \in C$.

(3) There exists a sequence $\left\{L_{n}\right\}$ of nonnegative real numbers such that

$$
\left\|T_{n} x-T_{n} y\right\| \leq L_{n}\|x-y\|, \quad \forall x, y \in C, \forall n \geq 0 .
$$

A discrete Lipschitzian semigroup $\mathcal{S}$ is called nonexpansive semigroup if $L_{n}=1$ for all $n \geq 0$, contraction semigroup if $0<L_{n}<1$ for all $n \geq 0$ and, asymptotically nonexpansive semigroup if $\limsup _{n \rightarrow \infty} L_{n} \leq 1$, respectively. We use $F(\mathcal{S})$ to denote the common fixed point set of the semigroup $\mathcal{S}$; that is, $F(\mathcal{S})=\left\{x \in C: T_{n} x=x, \forall n \geq 0\right\}$. 
Very recently, Kim [16] introduced asymptotically $\kappa$-SPC semigroup, a discrete family $\mathcal{S}=\left\{T_{n}: n \geq 0\right\}$ of self-mappings of $C$ which is said to be asymptotically $\kappa$-SPC semigroup on $C$ if, in addition to (1), (2) and the following condition ( $\left.3^{\prime}\right)$ are satisfied.

$\left(3^{\prime}\right)$ There exists a constant $\kappa \in[0,1)$ and a bounded sequence $\left\{L_{n}\right\}$ of nonnegative real numbers with $\lim \sup _{n \rightarrow \infty} L_{n} \leq 1$ such that

$$
\left\|T_{n} x-T_{n} y\right\|^{2} \leq L_{n}\|x-y\|^{2}+\kappa\left\|\left(I-T_{n}\right) x-\left(I-T_{n}\right) y\right\|^{2}, \forall x, y \in C, \forall n \geq 0 .
$$

Note that for both discrete asymptotically nonexpansive semigroups and discrete asymptotically $\kappa$-SPC semigroups, we can always assume that the Lipschitzian constants $\left\{L_{n}\right\}_{n \geq 0}$ are such that $L_{n} \geq 1$ for all $n \geq 0$ and $\lim _{n \rightarrow \infty} L_{n}=1$; otherwise, we replace $L_{n}$ for all $n \geq 0$ with $L_{n}^{\prime}=\max \left\{\sup _{m \geq n} L_{m}, 1\right\}$. Therefore, for a single asymptotically $\kappa$-SPC mapping $T: C \rightarrow C$ note that (1.15) immediately reduces to (1.9) by taking $T_{n} \equiv T^{n}$ and $\gamma_{n}=L_{n}-1$ such that $L_{n} \geq 1$ for all $n \geq 0$ and $\lim _{n \rightarrow \infty} L_{n}=1$.

To be more precise, Kim also showed in the framework of Hilbert spaces for the asymptotically $\kappa$-SPC semigroups that $T_{n}$ is continuous on $C$ for all $n \geq 0$ and that $F(S)$ is closed and convex (see Lemma 3.2 in [16]), and the demiclosedness principle (see Theorem 3.3 in [16]) holds in the sense that if $\left\{x_{n}\right\}$ is a sequence in $C$ such that $x_{n} \rightarrow z$ and limsup $\operatorname{sum}_{m \rightarrow \infty} \limsup _{n \rightarrow \infty}\left\|x_{n}-T_{m} x_{n}\right\|=0$ then, $\mathrm{z} \in F(\mathcal{S})=\bigcap_{n=0}^{\infty} F\left(T_{n}\right)$, and he also introduced an iterative scheme to find a common fixed point of a discrete asymptotically $\kappa$-SPC semigroup and a bounded sequence $\left\{L_{n}\right\} \subset[1, \infty)$ such that $\lim _{n \rightarrow \infty} L_{n}=1$ as follows:

$$
\begin{gathered}
x_{0}=x \in C \text { chosen arbitrarily, } \\
y_{n}=\alpha_{n} x_{n}+\left(1-\alpha_{n}\right) T_{n} x_{n}, \\
C_{n}=\left\{z \in C:\left\|y_{n}-z\right\|^{2} \leq\left\|x_{n}-z\right\|^{2}+\left(1-\alpha_{n}\right)\left(\theta_{n}+\left(\mathcal{\kappa}-\alpha_{n}\right)\left\|x_{n}-T_{n} x_{n}\right\|^{2}\right)\right\}, \\
Q_{n}=\left\{z \in C:\left\langle x_{n}-z, x_{0}-x_{n}\right\rangle \geq 0\right\}, \\
x_{n+1}=P_{C_{n} \cap Q_{n}}\left(x_{0}\right), \quad \forall n \in \mathbb{N} \cup\{0\},
\end{gathered}
$$

where $\theta_{n}=\left(L_{n}-1\right) \cdot \sup \left\{\left\|x_{n}-z\right\|^{2}: z \in F(S)\right\}<\infty$. He proved that under the parameter $0 \leq \alpha_{n}<1$ for all $n \in \mathbb{N} \cup\{0\}$, if $F(S)$ is a nonempty bounded subset of $C$, then the sequence $\left\{x_{n}\right\}$ generated by (1.16) converges strongly to $P_{F(\mathcal{S})}\left(x_{0}\right)$.

Inspired and motivated by the works mentioned above, in this paper, we introduce a general iterative scheme (3.1) below to find a common element of the set of common fixed point for a discrete asymptotically $\kappa$-SPC semigroup and the set of solutions of the mixed equilibrium problems in Hilbert spaces. The strong convergence theorem for the above two sets is obtained based on the shrinking projection method which extend and improve the corresponding ones due to Kim [16].

\section{Preliminaries}

Let $C$ be a nonempty closed convex subset of a real Hilbert space $H$. For a sequence $\left\{x_{n}\right\}$ in $H$, we denote the strong convergence and the weak convergence of $\left\{x_{n}\right\}$ to $x \in H$ by $x_{n} \rightarrow x$ and $x_{n} \rightarrow x$, respectively, and the weak $\omega$-limit set of $\left\{x_{n}\right\}$ by $\omega_{w}\left(x_{n}\right)=\left\{x: \exists x_{n_{j}} \rightarrow x\right\}$. 
For solving the mixed equilibrium problem, let us assume that the bifunction $\Phi: C \times$ $C \rightarrow \mathbb{R}$, the function $\varphi: C \rightarrow \mathbb{R} \cup\{+\infty\}$ and the set $C$ satisfy the following conditions.

(A1) $\Phi(x, x)=0$ for all $x \in C$.

(A2) $\Phi$ is monotone; that is, $\Phi(x, y)+\Phi(y, x) \leq 0$ for all $x, y \in C$.

(A3) For each $x, y, z \in C$,

$$
\lim _{t \downarrow 0} \Phi(t z+(1-t) x, y) \leq \Phi(x, y)
$$

(A4) For each $x \in C, y \mapsto \Phi(x, y)$ is convex and lower semicontinuous.

(A5) For each $y \in C, x \mapsto \Phi(x, y)$ is weakly upper semicontinuous.

(B1) For each $x \in C$ and $r>0$, there exists a bounded subset $D_{x} \subset C$ and $y_{x} \in C$ such that for any $z \in C \backslash D_{x}$,

$$
\Phi\left(z, y_{x}\right)+\varphi\left(y_{x}\right)-\varphi(z)+\frac{1}{r}\left\langle y_{x}-z, z-x\right\rangle<0
$$

(B2) $C$ is a bounded set.

Lemma 2.1 (see [17]). Let $H$ be a Hilbert space. For any $x, y \in H$ and $\lambda \in \mathbb{R}$, one has

$$
\|\lambda x+(1-\lambda) y\|^{2}=\lambda\|x\|^{2}+(1-\lambda)\|y\|^{2}-\lambda(1-\lambda)\|x-y\|^{2} .
$$

Lemma 2.2 (see [3]). Let C be a nonempty closed convex subset of a Hilbert space $H$. Then the following inequality holds:

$$
\left\langle x-P_{C} x, P_{C} x-y\right\rangle \geq 0, \quad \forall x \in H, y \in C .
$$

Lemma 2.3 (see [18]). Let $C$ be a nonempty closed convex subset of a Hilbert space $H, \Phi: C \times C \rightarrow$ $\mathbb{R}$ satisfying the conditions $(A 1)-(A 5)$, and let $\varphi: C \rightarrow \mathbb{R} \cup\{+\infty\}$ be a proper lower semicontinuous and convex function. Assume that either (B1) or (B2) holds. For $r>0$, define a mapping $S_{r}: C \rightarrow C$ as follows:

$$
S_{r}(x)=\left\{z \in C: \Phi(z, y)+\varphi(y)-\varphi(z)+\frac{1}{r}\langle y-z, z-x\rangle \geq 0, \forall y \in C\right\}
$$

for all $x \in C$. Then, the following statement hold.

(1) For each $x \in C, S_{r}(x) \neq \emptyset$.

(2) $S_{r}$ is single-valued.

(3) $S_{r}$ is firmly nonexpansive; that is, for any $x, y \in C$, 
ISRN Mathematical Analysis

$$
\left\|S_{r} x-S_{r} y\right\|^{2} \leq\left\langle S_{r} x-S_{r} y, x-y\right\rangle .
$$

(4) $F\left(S_{r}\right)=\operatorname{MEP}(\Phi, \varphi)$.

(5) $\operatorname{MEP}(\Phi, \varphi)$ is closed and convex.

Lemma 2.4 (see [3]). Every Hilbert space $H$ has Radon-Riesz property or Kadec-Klee property; that is, for a sequence $\left\{x_{n}\right\} \subset H$ with $x_{n} \rightarrow x$ and $\left\|x_{n}\right\| \rightarrow\|x\|$ then $x_{n} \rightarrow x$.

Lemma 2.5 (see [16]). Let $C$ be a nonempty closed convex subset of a Hilbert space $H$, and let $\mathcal{S}=\left\{T_{n}: n \geq 0\right\}$ be an asymptotically $\kappa$-strictly pseudocontractive semigroup on $C$. Let $\left\{x_{n}\right\}$ be a sequence in $C$ such that $\lim _{n \rightarrow \infty}\left\|x_{n}-x_{n+1}\right\|=0$ and $\lim _{n \rightarrow \infty}\left\|x_{n}-T_{n} x_{n}\right\|=0$. Then $\omega_{w}\left(x_{n}\right) \subset F(\mathcal{S})$.

\section{Main Results}

Theorem 3.1. Let $C$ be a nonempty closed convex subset of a real Hilbert space $H$, let $\Phi$ be a bifunction from $C \times C$ into $\mathbb{R}$ satisfying the conditions (A1)-(A5), and let $\varphi: C \rightarrow \mathbb{R} \cup\{+\infty\}$ be a proper lower semicontinuous and convex function with either (B1) or (B2) holds. Let $\mathcal{S}=\left\{T_{n}\right.$ : $n \geq 0\}$ be an asymptotically $\kappa$-SPC semigroup on $C$ for some $\kappa \in[0,1)$ and a bounded sequence $\left\{L_{n}\right\} \subset[1, \infty)$ such that $\lim _{n \rightarrow \infty} L_{n}=1$. Assume that $\Omega:=F(S) \cap \operatorname{MEP}(\Phi, \varphi)$ is a nonempty bounded subset of $C$. For $x_{0}=x \in C$ chosen arbitrarily, suppose that $\left\{x_{n}\right\},\left\{y_{n}\right\}$, and $\left\{u_{n}\right\}$ are generated iteratively by

$$
\begin{gathered}
u_{n} \in C \text { such that } \Phi\left(u_{n}, y\right)+\varphi(y)-\varphi\left(u_{n}\right)+\frac{1}{r_{n}}\left\langle y-u_{n}, u_{n}-x_{n}\right\rangle \geq 0, \quad \forall y \in C, \\
y_{n}=\alpha_{n} u_{n}+\left(1-\alpha_{n}\right) T_{n} u_{n} \\
C_{n+1}=\left\{z \in C_{n} \cap Q_{n}:\left\|y_{n}-z\right\|^{2} \leq\left\|x_{n}-z\right\|^{2}+\left(1-\alpha_{n}\right)\left(\theta_{n}+\left(\kappa-\alpha_{n}\right)\left\|u_{n}-T_{n} u_{n}\right\|^{2}\right)\right\}, \\
Q_{n+1}=\left\{z \in C_{n} \cap Q_{n}:\left\langle x_{n}-z, x_{0}-x_{n}\right\rangle \geq 0\right\}, \\
C_{0}=Q_{0}=C, \\
x_{n+1}=P_{C_{n+1} \cap Q_{n+1}}\left(x_{0}\right), \quad \forall n \in \mathbb{N} \cup\{0\},
\end{gathered}
$$

where $\theta_{n}=\left(L_{n}-1\right) \cdot \sup \left\{\left\|x_{n}-z\right\|^{2}: z \in \Omega\right\}<\infty$ satisfying the following conditions:

(C1) $\left\{\alpha_{n}\right\} \subset[a, b]$ such that $\kappa<a<b<1$,

(C2) $\left\{r_{n}\right\} \subset[r, \infty)$ for some $r>0$,

(C3) $\sum_{n=0}^{\infty}\left|r_{n+1}-r_{n}\right|<\infty$.

Then the sequences $\left\{x_{n}\right\},\left\{y_{n}\right\}$, and $\left\{u_{n}\right\}$ converge strongly to $w=P_{\Omega}\left(x_{0}\right)$.

Proof. Pick $p \in \Omega$. Therefore, by (3.1) and the definition of $S_{r_{n}}$ in Lemma 2.3, we have

$$
u_{n}=S_{r_{n}} x_{n} \in \operatorname{dom} \varphi
$$


and, by $F(\mathcal{S})=\bigcap_{n=0}^{\infty} F\left(T_{n}\right)$ and Lemma 2.3(4), we have

$$
T_{n} p=p=S_{r_{n}} p
$$

By (3.2), (3.3), and the nonexpansiveness of $S_{r_{n}}$, we have

$$
\left\|u_{n}-p\right\|=\left\|S_{r_{n}} x_{n}-S_{r_{n}} p\right\| \leq\left\|x_{n}-p\right\| .
$$

By (3.3), (3.4), Lemma 2.1, and the asymptotically $\kappa$-SPC semigroupness of $\mathcal{S}$, we have

$$
\begin{aligned}
\left\|y_{n}-p\right\|^{2} & =\left\|\alpha_{n}\left(u_{n}-p\right)+\left(1-\alpha_{n}\right)\left(T_{n} u_{n}-p\right)\right\|^{2} \\
& =\alpha_{n}\left\|u_{n}-p\right\|^{2}+\left(1-\alpha_{n}\right)\left\|T_{n} u_{n}-p\right\|^{2}-\alpha_{n}\left(1-\alpha_{n}\right)\left\|u_{n}-T_{n} u_{n}\right\|^{2} \\
& \leq \alpha_{n}\left\|u_{n}-p\right\|^{2}+\left(1-\alpha_{n}\right)\left(L_{n}\left\|u_{n}-p\right\|^{2}+\kappa\left\|u_{n}-T_{n} u_{n}\right\|^{2}\right)-\alpha_{n}\left(1-\alpha_{n}\right)\left\|u_{n}-T_{n} u_{n}\right\|^{2} \\
& =\left(1+\left(1-\alpha_{n}\right)\left(L_{n}-1\right)\right)\left\|u_{n}-p\right\|^{2}+\left(1-\alpha_{n}\right)\left(\kappa-\alpha_{n}\right)\left\|u_{n}-T_{n} u_{n}\right\|^{2} \\
& \leq\left\|x_{n}-p\right\|^{2}+\left(1-\alpha_{n}\right)\left(\theta_{n}+\left(\kappa-\alpha_{n}\right)\left\|u_{n}-T_{n} u_{n}\right\|^{2}\right),
\end{aligned}
$$

where $\theta_{n}:=\left(L_{n}-1\right) \cdot \sup \left\{\left\|x_{n}-z\right\|^{2}: z \in \Omega\right\}$ for all $n \in \mathbb{N} \cup\{0\}$.

Firstly, we prove that $C_{n} \cap Q_{n}$ is closed and convex for all $n \in \mathbb{N} \cup\{0\}$. It is obvious that $C_{0} \cap Q_{0}$ is closed and by mathematical induction that $C_{n} \cap Q_{n}$ is closed for all $n \geq 1$; that is $C_{n} \cap Q_{n}$ is closed for all $n \in \mathbb{N} \cup\{0\}$. Let $\epsilon_{n}=\left(1-\alpha_{n}\right)\left(\theta_{n}+\left(k-\alpha_{n}\right)\left\|u_{n}-T_{n} u_{n}\right\|^{2}\right)$ since, for any $z \in C,\left\|y_{n}-z\right\|^{2} \leq\left\|x_{n}-z\right\|^{2}+\epsilon_{n}$ is equivalent to

$$
\left\|y_{n}-x_{n}\right\|^{2}+2\left\langle y_{n}-x_{n}, x_{n}-z\right\rangle-\epsilon_{n} \leq 0,
$$

for all $n \in \mathbb{N} \cup\{0\}$. Therefore, for any $z_{1}, z_{2} \in C_{n+1} \cap Q_{n+1} \subset C_{n} \cap Q_{n}$ and $\epsilon \in(0,1)$, we have

$$
\begin{aligned}
\left\|y_{n}-x_{n}\right\|^{2}+2\left\langle y_{n}-x_{n}, x_{n}-\left(\epsilon z_{1}+(1-\epsilon) z_{2}\right)\right\rangle-\epsilon_{n}= & \epsilon\left(\left\|y_{n}-x_{n}\right\|^{2}+2\left\langle y_{n}-x_{n}, x_{n}-z_{1}\right\rangle-\epsilon_{n}\right)+(1-\epsilon) \\
& \times\left(\left\|y_{n}-x_{n}\right\|^{2}+2\left\langle y_{n}-x_{n}, x_{n}-z_{2}\right\rangle-\epsilon_{n}\right) \\
\leq 0 &
\end{aligned}
$$

for all $n \in \mathbb{N} \cup\{0\}$, and we have

$$
\begin{aligned}
\left\langle x_{n}-\left(\epsilon z_{1}+(1-\epsilon) z_{2}\right), x_{0}-x_{n}\right\rangle & =\epsilon\left\langle x_{n}-z_{1}, x_{0}-x_{n}\right\rangle+(1-\epsilon)\left\langle x_{n}-z_{2}, x_{0}-x_{n}\right\rangle \\
& \geq 0
\end{aligned}
$$


for all $n \in \mathbb{N} \cup\{0\}$. Since $C_{0} \cap Q_{0}$ is convex and by putting $n=0$ in (3.6), (3.7), and (3.8), we have that $C_{1} \cap Q_{1}$ is convex. Suppose that $x_{k}$ is given and $C_{k} \cap Q_{k}$ is convex for some $k \geq 1$. It follows by putting $n=k$ in (3.6), (3.7), and (3.8) that $C_{k+1} \cap Q_{k+1}$ is convex. Therefore, by mathematical induction, we have that $C_{n} \cap Q_{n}$ is convex for all $n \geq 1$; that is, $C_{n} \cap Q_{n}$ is convex for all $n \in \mathbb{N} \cup\{0\}$. Hence, we obtain that $C_{n} \cap Q_{n}$ is closed and convex for all $n \in \mathbb{N} \cup\{0\}$.

Next, we prove that $\Omega \subset C_{n} \cap Q_{n}$ for all $n \in \mathbb{N} \cup\{0\}$. It is obvious that $p \in \Omega \subset C=$ $C_{0} \cap Q_{0}$. Therefore, by (3.1) and (3.5), we have $p \in C_{1}$ and note that $p \in C=Q_{1}$, and so $p \in C_{1} \cap Q_{1}$. Hence, we have $\Omega \subset C_{1} \cap Q_{1}$. Since $C_{1} \cap Q_{1}$ is a nonempty closed convex subset of $C$, there exists a unique element $x_{1} \in C_{1} \cap Q_{1}$ such that $x_{1}=P_{C_{1} \cap Q_{1}}\left(x_{0}\right)$. Suppose that $x_{k} \in C_{k} \cap Q_{k}$ is given such that $x_{k}=P_{C_{k} \cap Q_{k}}\left(x_{0}\right)$ and $p \in \Omega \subset C_{k} \cap Q_{k}$ for some $k \geq 1$. Therefore, by (3.1) and (3.5), we have $p \in C_{k+1}$. Since $x_{k}=P_{C_{k} \cap Q_{k}}\left(x_{0}\right)$; therefore, by Lemma 2.2, we have

$$
\left\langle x_{k}-z, x_{0}-x_{k}\right\rangle \geq 0
$$

for all $z \in C_{k} \cap Q_{k}$. Thus, by (3.1), we have $p \in Q_{k+1}$, and so $p \in C_{k+1} \cap Q_{k+1}$. Hence, we have $\Omega \subset C_{k+1} \cap Q_{k+1}$. Since $C_{k+1} \cap Q_{k+1}$ is a nonempty closed convex subset of $C$, there exists a unique element $x_{k+1} \in C_{k+1} \cap Q_{k+1}$ such that $x_{k+1}=P_{C_{k+1} \cap Q_{k+1}}\left(x_{0}\right)$. Therefore, by mathematical induction, we obtain $\Omega \subset C_{n} \cap Q_{n}$ for all $n \geq 1$, and so $\Omega \subset C_{n} \cap Q_{n}$ for all $n \in \mathbb{N} \cup\{0\}$, and we can define $x_{n+1}=P_{C_{n+1} \cap Q_{n+1}}\left(x_{0}\right)$ for all $n \in \mathbb{N} \cup\{0\}$. Hence, we obtain that the iteration (3.1) is well defined.

Next, we prove that $\left\{x_{n}\right\}$ is bounded. Since $x_{n}=P_{C_{n} \cap Q_{n}}\left(x_{0}\right)$ for all $n \in \mathbb{N} \cup\{0\}$, we have

$$
\left\|x_{n}-x_{0}\right\| \leq\left\|z-x_{0}\right\|
$$

for all $z \in C_{n} \cap Q_{n}$. It follows by $p \in \Omega \subset C_{n} \cap Q_{n}$ that $\left\|x_{n}-x_{0}\right\| \leq\left\|p-x_{0}\right\|$ for all $n \in \mathbb{N} \cup\{0\}$. This implies that $\left\{x_{n}\right\}$ is bounded and so are $\left\{y_{n}\right\}$ and $\left\{u_{n}\right\}$.

Next, we prove that $\left\|x_{n}-x_{n+1}\right\| \rightarrow 0$ and $\left\|u_{n}-u_{n+1}\right\| \rightarrow 0$ as $n \rightarrow \infty$. Since $x_{n+1}=$ $P_{C_{n+1} \cap Q_{n+1}}\left(x_{0}\right) \in C_{n+1} \cap Q_{n+1} \subset C_{n} \cap Q_{n}$; therefore, by (3.10), we have $\left\|x_{n}-x_{0}\right\| \leq\left\|x_{n+1}-x_{0}\right\|$ for all $n \in \mathbb{N} \cup\{0\}$. This implies that $\left\{\left\|x_{n}-x_{0}\right\|\right\}$ is a bounded nondecreasing sequence; there exists the limit of $\left\|x_{n}-x_{0}\right\|$; that is,

$$
\lim _{n \rightarrow \infty}\left\|x_{n}-x_{0}\right\|=m
$$

for some $m \geq 0$. Since $x_{n+1} \in Q_{n+1}$; therefore, by (3.1), we have

$$
\left\langle x_{n}-x_{n+1}, x_{0}-x_{n}\right\rangle \geq 0 .
$$

It follows by (3.12) that

$$
\begin{aligned}
\left\|x_{n}-x_{n+1}\right\|^{2}= & \left\|\left(x_{n}-x_{0}\right)+\left(x_{0}-x_{n+1}\right)\right\|^{2} \\
= & \left\|x_{n}-x_{0}\right\|^{2}+2\left\langle x_{n}-x_{0}, x_{0}-x_{n}\right\rangle \\
& +2\left\langle x_{n}-x_{0}, x_{n}-x_{n+1}\right\rangle+\left\|x_{n+1}-x_{0}\right\|^{2} \\
\leq & \left\|x_{n+1}-x_{0}\right\|^{2}-\left\|x_{n}-x_{0}\right\|^{2} .
\end{aligned}
$$


Therefore, by (3.11), we obtain

$$
\left\|x_{n}-x_{n+1}\right\| \longrightarrow 0 \text { as } n \longrightarrow \infty
$$

Indeed, from (3.1), we have

$$
\begin{gathered}
\Phi\left(u_{n}, y\right)+\varphi(y)-\varphi\left(u_{n}\right)+\frac{1}{r_{n}}\left\langle y-u_{n}, u_{n}-x_{n}\right\rangle \geq 0, \quad \forall y \in C, \\
\Phi\left(u_{n+1}, y\right)+\varphi(y)-\varphi\left(u_{n+1}\right)+\frac{1}{r_{n+1}}\left\langle y-u_{n+1}, u_{n+1}-x_{n+1}\right\rangle \geq 0, \quad \forall y \in C .
\end{gathered}
$$

Substituting $y=u_{n+1}$ into (3.15) and $y=u_{n}$ into (3.16), we have

$$
\begin{gathered}
\Phi\left(u_{n}, u_{n+1}\right)+\varphi\left(u_{n+1}\right)-\varphi\left(u_{n}\right)+\frac{1}{r_{n}}\left\langle u_{n+1}-u_{n}, u_{n}-x_{n}\right\rangle \geq 0 \\
\Phi\left(u_{n+1}, u_{n}\right)+\varphi\left(u_{n}\right)-\varphi\left(u_{n+1}\right)+\frac{1}{r_{n+1}}\left\langle u_{n}-u_{n+1}, u_{n+1}-x_{n+1}\right\rangle \geq 0 .
\end{gathered}
$$

Therefore, by the condition (A2), we get

$$
\begin{aligned}
0 & \leq \Phi\left(u_{n}, u_{n+1}\right)+\Phi\left(u_{n+1}, u_{n}\right)+\left\langle u_{n+1}-u_{n}, \frac{u_{n}-x_{n}}{r_{n}}-\frac{u_{n+1}-x_{n+1}}{r_{n+1}}\right\rangle \\
& \leq\left\langle u_{n+1}-u_{n}, \frac{u_{n}-x_{n}}{r_{n}}-\frac{u_{n+1}-x_{n+1}}{r_{n+1}}\right\rangle .
\end{aligned}
$$

It follows that

$$
\begin{aligned}
0 & \leq\left\langle u_{n+1}-u_{n},\left(u_{n}-u_{n+1}\right)+\left(u_{n+1}-x_{n}\right)-\frac{r_{n}}{r_{n+1}}\left(u_{n+1}-x_{n+1}\right)\right\rangle \\
& =\left\langle u_{n+1}-u_{n}, u_{n}-u_{n+1}\right\rangle+\left\langle u_{n+1}-u_{n}\left(u_{n+1}-x_{n+1}\right)+\left(x_{n+1}-x_{n}\right)-\frac{r_{n}}{r_{n+1}}\left(u_{n+1}-x_{n+1}\right)\right\rangle .
\end{aligned}
$$

Thus, we have

$$
\begin{aligned}
\left\|u_{n+1}-u_{n}\right\|^{2} & \leq\left\langle u_{n+1}-u_{n},\left(x_{n+1}-x_{n}\right)+\left(1-\frac{r_{n}}{r_{n+1}}\right)\left(u_{n+1}-x_{n+1}\right)\right\rangle \\
& \leq\left\|u_{n+1}-u_{n}\right\|\left(\left\|x_{n+1}-x_{n}\right\|+\left|1-\frac{r_{n}}{r_{n+1}}\right|\left\|u_{n+1}-x_{n+1}\right\|\right) .
\end{aligned}
$$


It follows by the condition (C2) that

$$
\begin{aligned}
\left\|u_{n+1}-u_{n}\right\| & \leq\left\|x_{n+1}-x_{n}\right\|+\frac{\left|r_{n+1}-r_{n}\right|}{r_{n+1}}\left\|u_{n+1}-x_{n+1}\right\| \\
& \leq\left\|x_{n+1}-x_{n}\right\|+\left(\frac{L}{r}\right)\left|r_{n+1}-r_{n}\right|
\end{aligned}
$$

where $L=\sup _{n \geq 0}\left\|u_{n}-x_{n}\right\|<\infty$. Therefore, by the condition (C3) and (3.14), we obtain

$$
\left\|u_{n}-u_{n+1}\right\| \longrightarrow 0 \text { as } n \longrightarrow \infty
$$

Next, we prove that $\left\|u_{n}-T_{n} u_{n}\right\| \rightarrow 0,\left\|y_{n}-x_{n}\right\| \rightarrow 0$, and $\left\|u_{n}-x_{n}\right\| \rightarrow 0$ as $n \rightarrow \infty$. Since $x_{n+1} \in C_{n+1}$, by (3.1), we have

$$
\left\|y_{n}-x_{n+1}\right\|^{2} \leq\left\|x_{n}-x_{n+1}\right\|^{2}+\left(1-\alpha_{n}\right)\left(\theta_{n}+\left(\kappa-\alpha_{n}\right)\left\|u_{n}-T_{n} u_{n}\right\|^{2}\right) .
$$

It follows that

$$
\begin{aligned}
\left(1-\alpha_{n}\right)\left(\alpha_{n}-\kappa\right)\left\|u_{n}-T_{n} u_{n}\right\|^{2} & \leq\left\|x_{n}-x_{n+1}\right\|^{2}+\left(1-\alpha_{n}\right) \theta_{n}-\left\|y_{n}-x_{n+1}\right\|^{2} \\
& \leq\left\|x_{n}-x_{n+1}\right\|^{2}+\theta_{n} .
\end{aligned}
$$

Therefore, by the condition (C1), (3.14), and $\lim _{n \rightarrow \infty} \theta_{n}=0$, we obtain

$$
\left\|u_{n}-T_{n} u_{n}\right\| \longrightarrow 0 \quad \text { as } n \longrightarrow \infty
$$

From (3.23) and the condition (C1), we have

$$
\left\|y_{n}-x_{n+1}\right\|^{2} \leq\left\|x_{n}-x_{n+1}\right\|^{2}+\theta_{n}
$$

Therefore,

$$
\begin{aligned}
\left\|y_{n}-x_{n}\right\|^{2} & =\left\|\left(y_{n}-x_{n+1}\right)+\left(x_{n+1}-x_{n}\right)\right\|^{2} \\
& =\left\|y_{n}-x_{n+1}\right\|^{2}+2\left\langle y_{n}-x_{n+1}, x_{n+1}-x_{n}\right\rangle+\left\|x_{n+1}-x_{n}\right\|^{2} \\
& \leq\left\|y_{n}-x_{n+1}\right\|^{2}+2\left\|y_{n}-x_{n+1}\right\|\left\|x_{n+1}-x_{n}\right\|+\left\|x_{n+1}-x_{n}\right\|^{2} \\
& \leq 2\left\|x_{n+1}-x_{n}\right\|\left(\left\|x_{n+1}-x_{n}\right\|+\left\|y_{n}-x_{n+1}\right\|\right)+\theta_{n} .
\end{aligned}
$$

Hence, by (3.14) and $\lim _{n \rightarrow \infty} \theta_{n}=0$, we obtain

$$
\left\|y_{n}-x_{n}\right\| \longrightarrow 0 \text { as } n \longrightarrow \infty
$$


By (3.2), (3.3), and the firmly nonexpansiveness of $S_{r_{n}}$, we have

$$
\begin{aligned}
\left\|u_{n}-p\right\|^{2} & \leq\left\langle S_{r_{n}} x_{n}-S_{r_{n}} p, x_{n}-p\right\rangle=\left\langle u_{n}-p, x_{n}-p\right\rangle \\
& =\frac{1}{2}\left(\left\|u_{n}-p\right\|^{2}+\left\|x_{n}-p\right\|^{2}-\left\|u_{n}-x_{n}\right\|^{2}\right) .
\end{aligned}
$$

It follows that

$$
\left\|u_{n}-p\right\|^{2} \leq\left\|x_{n}-p\right\|^{2}-\left\|u_{n}-x_{n}\right\|^{2}
$$

Therefore, by the condition (C1) and (3.5), we have

$$
\begin{aligned}
\left\|y_{n}-p\right\|^{2} & \leq\left(1+\left(1-\alpha_{n}\right)\left(L_{n}-1\right)\right)\left\|u_{n}-p\right\|^{2}+\left(1-\alpha_{n}\right)\left(\kappa-\alpha_{n}\right)\left\|u_{n}-T_{n} u_{n}\right\|^{2} \\
& \leq\left(L_{n}-\alpha_{n}\left(L_{n}-1\right)\right)\left\|u_{n}-p\right\|^{2} \leq L_{n}\left\|u_{n}-p\right\|^{2} \\
& \leq L_{n}\left(\left\|x_{n}-p\right\|^{2}-\left\|u_{n}-x_{n}\right\|^{2}\right) .
\end{aligned}
$$

It follows that

$$
\begin{aligned}
L_{n}\left\|u_{n}-x_{n}\right\|^{2} & \leq L_{n}\left\|x_{n}-p\right\|^{2}-\left\|y_{n}-p\right\|^{2} \\
& =\left(L_{n}-1\right)\left\|x_{n}-p\right\|^{2}+\left\|x_{n}-p\right\|^{2}-\left\|y_{n}-p\right\|^{2} \\
& \leq \theta_{n}+\left\|x_{n}-y_{n}\right\|\left(\left\|x_{n}-p\right\|+\left\|y_{n}-p\right\|\right) .
\end{aligned}
$$

Hence, by (3.28) and $\lim _{n \rightarrow \infty} \theta_{n}=0$, we obtain

$$
\left\|u_{n}-x_{n}\right\| \longrightarrow 0 \text { as } n \longrightarrow \infty
$$

Since $\left\{u_{n}\right\}$ is bounded, there exists a subsequence $\left\{u_{n_{i}}\right\}$ of $\left\{u_{n}\right\}$ which converges weakly to $\bar{w}$. Next, we prove that $\bar{w} \in \Omega$. From (3.22) and (3.25), we have $\left\|u_{n_{i}}-u_{n_{i}+1}\right\| \rightarrow 0$, and $\left\|u_{n_{i}}-T_{n_{i}} u_{n_{i}}\right\| \rightarrow 0$ as $i \rightarrow \infty$; therefore, by Lemma 2.5, we obtain $\bar{w} \in F(\mathcal{S})$. From (3.1), we have

$$
0 \leq \Phi\left(u_{n}, y\right)+\varphi(y)-\varphi\left(u_{n}\right)+\frac{1}{r_{n}}\left\langle y-u_{n}, u_{n}-x_{n}\right\rangle, \quad \forall y \in C
$$

It follows by the condition (A2) that

$$
\begin{aligned}
\Phi\left(y, u_{n}\right) & \leq \Phi\left(y, u_{n}\right)+\Phi\left(u_{n}, y\right)+\varphi(y)-\varphi\left(u_{n}\right)+\frac{1}{r_{n}}\left\langle y-u_{n}, u_{n}-x_{n}\right\rangle, \quad \forall y \in C \\
& \leq \varphi(y)-\varphi\left(u_{n}\right)+\frac{1}{r_{n}}\left\langle y-u_{n}, u_{n}-x_{n}\right\rangle, \quad \forall y \in C .
\end{aligned}
$$


Hence,

$$
\varphi(y)-\varphi\left(u_{n_{i}}\right)+\left\langle y-u_{n_{i}}, \frac{u_{n_{i}}-x_{n_{i}}}{r_{n_{i}}}\right\rangle \geq \Phi\left(y, u_{n_{i}}\right), \quad \forall y \in C
$$

Therefore, from (3.33) and by $u_{n_{i}} \rightarrow \bar{w}$ as $i \rightarrow \infty$, we obtain

$$
\Phi(y, \bar{w})+\varphi(\bar{w})-\varphi(y) \leq 0, \quad \forall y \in C
$$

For a constant $t$ with $0<t<1$ and $y \in C$, let $y_{t}=t y+(1-t) \bar{w}$. Since $y, \bar{w} \in C$, thus, $y_{t} \in C$. So, from (3.37), we have

$$
\Phi\left(y_{t}, \bar{w}\right)+\varphi(\bar{w})-\varphi\left(y_{t}\right) \leq 0
$$

By (3.38), the conditions (A1) and (A4), and the convexity of $\varphi$, we have

$$
\begin{aligned}
0 & =\Phi\left(y_{t}, y_{t}\right)+\varphi\left(y_{t}\right)-\varphi\left(y_{t}\right) \\
& \leq\left(t \Phi\left(y_{t}, y\right)+(1-t) \Phi\left(y_{t}, \bar{w}\right)\right)+(t \varphi(y)+(1-t) \varphi(\bar{w}))-\varphi\left(y_{t}\right) \\
& =t\left(\Phi\left(y_{t}, y\right)+\varphi(y)-\varphi\left(y_{t}\right)\right)+(1-t)\left(\Phi\left(y_{t}, \bar{w}\right)+\varphi(\bar{w})-\varphi\left(y_{t}\right)\right) \\
& \leq t\left(\Phi\left(y_{t}, y\right)+\varphi(y)-\varphi\left(y_{t}\right)\right) .
\end{aligned}
$$

It follows that

$$
\Phi\left(y_{t}, y\right)+\varphi(y)-\varphi\left(y_{t}\right) \geq 0
$$

Therefore, by the condition (A3) and the weakly lower semicontinuity of $\varphi$, we have $\Phi(\bar{w}, y)+\varphi(y)-\varphi(\bar{w}) \geq 0$ as $t \rightarrow 0$ for all $y \in C$, and; hence, we obtain $\bar{w} \in \operatorname{MEP}(\Phi, \varphi)$, and so $\bar{w} \in \Omega$.

Since $\Omega$ is a nonempty closed convex subset of $C$, there exists a unique $w \in \Omega$ such that $w=P_{\Omega}\left(x_{0}\right)$. Next, we prove that $x_{n} \rightarrow w$ as $n \rightarrow \infty$. Since $w=P_{\Omega}\left(x_{0}\right)$, we have $\left\|x_{0}-w\right\| \leq\left\|x_{0}-z\right\|$ for all $z \in \Omega$; it follows that

$$
\left\|x_{0}-w\right\| \leq\left\|x_{0}-\bar{w}\right\|
$$

Since $w \in \Omega \subset C_{n} \cap Q_{n}$; therefore, by (3.10), we have

$$
\left\|x_{0}-x_{n}\right\| \leq\left\|x_{0}-w\right\|
$$

Since $\left\|x_{n_{i}}-u_{n_{\mathrm{i}}}\right\| \rightarrow 0$ by (3.33) and $u_{n_{i}} \rightarrow \bar{w}$, we have $x_{n_{i}} \rightarrow \bar{w}$ as $i \rightarrow \infty$. Therefore, by (3.41), (3.42), and the weak lower semicontinuity of norm, we have

$$
\left\|x_{0}-w\right\| \leq\left\|x_{0}-\bar{w}\right\| \leq \liminf _{i \rightarrow \infty}\left\|x_{0}-x_{n_{i}}\right\| \leq \limsup _{i \rightarrow \infty}\left\|x_{0}-x_{n_{i}}\right\| \leq\left\|x_{0}-w\right\| .
$$


It follows that

$$
\left\|x_{0}-w\right\|=\lim _{i \rightarrow \infty}\left\|x_{0}-x_{n_{i}}\right\|=\left\|x_{0}-\bar{w}\right\|
$$

Since $x_{n_{i}} \rightarrow \bar{w}$ as $i \rightarrow \infty$; therefore, we have

$$
\left(x_{0}-x_{n_{i}}\right) \rightarrow\left(x_{0}-\bar{w}\right) \quad \text { as } i \longrightarrow \infty
$$

Hence, from (3.44), (3.45), the Kadec-Klee property, and the uniqueness of $w=P_{\Omega}\left(x_{0}\right)$, we obtain

$$
x_{n_{i}} \longrightarrow \bar{w}=w \quad \text { as } i \longrightarrow \infty .
$$

It follows that $\left\{x_{n}\right\}$ converges strongly to $w$ and so are $\left\{y_{n}\right\}$ and $\left\{u_{n}\right\}$. This completes the proof.

Remark 3.2. The iteration (3.1) is the difference with the iterative scheme of Kim [16] as the following.

(1) The sequence $\left\{x_{n}\right\}$ is a projection sequence of $x_{0}$ onto $C_{n} \cap Q_{n}$ for all $n \in \mathbb{N} \cup\{0\}$ such that

$$
C_{0} \cap Q_{0} \supset C_{1} \cap Q_{1} \supset \cdots \supset C_{n} \cap Q_{n} \supset \cdots \supset \Omega
$$

(2) A solving of a common element of the set of common fixed point for a discrete asymptotically $\kappa$-SPC semigroup and the set of solutions of the mixed equilibrium problems by iteration is obtained.

For solving the equilibrium problem, let us define the condition (B3) as the condition (B1) such that $\varphi=0$. We have the following result.

Corollary 3.3. Let $C$ be a nonempty closed convex subset of a real Hilbert space $H$, and let $\Phi$ be a bifunction from $C \times C$ into $\mathbb{R}$ satisfying the conditions (A1)-(A5) with either (B2) or (B3) holds. Let $\mathcal{S}=\left\{T_{n}: n \geq 0\right\}$ be an asymptotically $\kappa$-SPC semigroup on $C$ for some $\kappa \in[0,1)$ and a bounded sequence $\left\{L_{n}\right\} \subset[1, \infty)$ such that $\lim _{n \rightarrow \infty} L_{n}=1$. Assume that $\Omega:=F(\mathcal{S}) \cap E P(\Phi)$ is a nonempty bounded subset of C. For $x_{0}=x \in C$ chosen arbitrarily, suppose that $\left\{x_{n}\right\},\left\{y_{n}\right\}$, and $\left\{u_{n}\right\}$ are generated iteratively by

$$
\begin{gathered}
u_{n} \in C \text { such that } \Phi\left(u_{n}, y\right)+\frac{1}{r_{n}}\left\langle y-u_{n}, u_{n}-x_{n}\right\rangle \geq 0, \quad \forall y \in C, \\
y_{n}=\alpha_{n} u_{n}+\left(1-\alpha_{n}\right) T_{n} u_{n}, \\
C_{n+1}=\left\{z \in C_{n} \cap Q_{n}:\left\|y_{n}-z\right\|^{2} \leq\left\|x_{n}-z\right\|^{2}+\left(1-\alpha_{n}\right)\left(\theta_{n}+\left(\kappa-\alpha_{n}\right)\left\|u_{n}-T_{n} u_{n}\right\|^{2}\right)\right\}, \\
Q_{n+1}=\left\{z \in C_{n} \cap Q_{n}:\left\langle x_{n}-z, x_{0}-x_{n}\right\rangle \geq 0\right\},
\end{gathered}
$$


ISRN Mathematical Analysis

$$
\begin{gathered}
C_{0}=Q_{0}=C, \\
x_{n+1}=P_{C_{n+1} \cap Q_{n+1}}\left(x_{0}\right), \quad \forall n \in \mathbb{N} \cup\{0\},
\end{gathered}
$$

where $\theta_{n}=\left(L_{n}-1\right) \cdot \sup \left\{\left\|x_{n}-z\right\|^{2}: z \in \Omega\right\}<\infty$ satisfying the following conditions:

(C1) $\left\{\alpha_{n}\right\} \subset[a, b]$ such that $\kappa<a<b<1$,

(C2) $\left\{r_{n}\right\} \subset[r, \infty)$ for some $r>0$,

(C3) $\sum_{n=0}^{\infty}\left|r_{n+1}-r_{n}\right|<\infty$.

Then the sequences $\left\{x_{n}\right\},\left\{y_{n}\right\}$, and $\left\{u_{n}\right\}$ converge strongly to $w=P_{\Omega}\left(x_{0}\right)$.

Proof. It is concluded from Theorem 3.1 immediately, by putting $\varphi=0$.

Corollary 3.4. Let $C$ be a nonempty closed convex subset of a real Hilbert space $H$. Let $\mathcal{S}=\left\{T_{n}\right.$ : $n \geq 0\}$ be an asymptotically $\kappa$-SPC semigroup on $C$ for some $\kappa \in[0,1)$ and a bounded sequence $\left\{L_{n}\right\} \subset[1, \infty)$ such that $\lim _{n \rightarrow \infty} L_{n}=1$. Assume that $F(S)$ is a nonempty bounded subset of $C$. For $x_{0}=x \in C$ chosen arbitrarily, suppose that $\left\{x_{n}\right\}$ and $\left\{y_{n}\right\}$ are generated iteratively by

$$
\begin{gathered}
y_{n}=\alpha_{n} x_{n}+\left(1-\alpha_{n}\right) T_{n} x_{n}, \\
C_{n+1}=\left\{z \in C_{n} \cap Q_{n}:\left\|y_{n}-z\right\|^{2} \leq\left\|x_{n}-z\right\|^{2}+\left(1-\alpha_{n}\right)\left(\theta_{n}+\left(\kappa-\alpha_{n}\right)\left\|x_{n}-T_{n} x_{n}\right\|^{2}\right)\right\}, \\
Q_{n+1}=\left\{z \in C_{n} \cap Q_{n}:\left\langle x_{n}-z, x_{0}-x_{n}\right\rangle \geq 0\right\}, \\
C_{0}=Q_{0}=C, \\
x_{n+1}=P_{C_{n+1} \cap Q_{n+1}}\left(x_{0}\right), \quad \forall n \in \mathbb{N} \cup\{0\},
\end{gathered}
$$

where $\theta_{n}=\left(L_{n}-1\right) \cdot \sup \left\{\left\|x_{n}-z\right\|^{2}: z \in F(\mathcal{S})\right\}<\infty$ and $\left\{\alpha_{n}\right\} \subset[a, b]$ such that $\kappa<a<b<1$. Then the sequences $\left\{x_{n}\right\}$ and $\left\{y_{n}\right\}$ converge strongly to $w=P_{F(S)}\left(x_{0}\right)$.

Proof. It is concluded from Corollary 3.3 immediately, by putting $\Phi=0$.

\section{Applications}

We introduce the equilibrium problem to the optimization problem:

$$
\min _{x \in C} \zeta(x)
$$

where $C$ is a nonempty closed convex subset of a real Hilbert space $H$ and $\zeta: C \rightarrow \mathbb{R} \cup\{+\infty\}$ is a proper convex and lower semicontinuous. We denote by $\operatorname{Argmin}(\zeta)$ the set of solutions of problem (4.1). We define the condition (B4) as the condition (B3) such that $\Phi: C \times C \rightarrow \mathbb{R}$ is a bifunction defined by $\Phi(x, y)=\zeta(y)-\zeta(x)$ for all $x, y \in C$. Observe that $E P(\Phi)=\operatorname{Argmin}(\zeta)$. We give the interesting result as the following theorem. 
Theorem 4.1. Let $C$ be a nonempty closed convex subset of a real Hilbert space $H$, and let $\zeta: C \rightarrow$ $\mathbb{R} \cup\{+\infty\}$ be a proper lower semicontinuous and convex function with either (B2) or (B4) holds. Let $\mathcal{S}=\left\{T_{n}: n \geq 0\right\}$ be an asymptotically $\kappa$-SPC semigroup on $C$ for some $\mathcal{\kappa} \in[0,1)$ and a bounded sequence $\left\{L_{n}\right\} \subset[1, \infty)$ such that $\lim _{n \rightarrow \infty} L_{n}=1$. Assume that $\Omega:=F(\mathcal{S}) \cap \operatorname{Argmin}(\zeta)$ is a nonempty bounded subset of $C$. For $x_{0}=x \in C$ chosen arbitrarily, suppose that $\left\{x_{n}\right\},\left\{y_{n}\right\}$ and $\left\{u_{n}\right\}$ are generated iteratively by

$$
\begin{gathered}
u_{n} \in C \text { such that } \zeta(y)-\zeta\left(u_{n}\right)+\frac{1}{r_{n}}\left\langle y-u_{n}, u_{n}-x_{n}\right\rangle \geq 0, \quad \forall y \in C, \\
y_{n}=\alpha_{n} u_{n}+\left(1-\alpha_{n}\right) T_{n} u_{n}, \\
C_{n+1}=\left\{z \in C_{n} \cap Q_{n}:\left\|y_{n}-z\right\|^{2} \leq\left\|x_{n}-z\right\|^{2}+\left(1-\alpha_{n}\right)\left(\theta_{n}+\left(\mathcal{k}-\alpha_{n}\right)\left\|u_{n}-T_{n} u_{n}\right\|^{2}\right)\right\}, \\
Q_{n+1}=\left\{z \in C_{n} \cap Q_{n}:\left\langle x_{n}-z, x_{0}-x_{n}\right\rangle \geq 0\right\}, \\
C_{0}=Q_{0}=C, \\
x_{n+1}=P_{C_{n+1} \cap Q_{n+1}}\left(x_{0}\right), \quad \forall n \in \mathbb{N} \cup\{0\},
\end{gathered}
$$

where $\theta_{n}=\left(L_{n}-1\right) \cdot \sup \left\{\left\|x_{n}-z\right\|^{2}: z \in \Omega\right\}<\infty$ satisfying the following conditions:

(C1) $\left\{\alpha_{n}\right\} \subset[a, b]$ such that $\kappa<a<b<1$,

(C2) $\left\{r_{n}\right\} \subset[r, \infty)$ for some $r>0$,

(C3) $\sum_{n=0}^{\infty}\left|r_{n+1}-r_{n}\right|<\infty$.

Then the sequences $\left\{x_{n}\right\},\left\{y_{n}\right\}$, and $\left\{u_{n}\right\}$ converge strongly to $w=P_{\Omega}\left(x_{0}\right)$.

Proof. It is concluded from Corollary 3.3 immediately, by defining $\Phi(x, y)=\zeta(y)-\zeta(x)$ for all $x, y \in C$.

\section{References}

[1] E. Blum and W. Oettli, "From optimization and variational inequalities to equilibrium problems," The Mathematics Student, vol. 63, no. 1-4, pp. 123-145, 1994.

[2] P. Hartman and G. Stampacchia, "On some non-linear elliptic differential-functional equations," Acta Mathematica, vol. 115, pp. 271-310, 1966.

[3] W. Takahashi, Nonlinear Functional Analysis, Yokohama Publishers, Yokohama, Japan, 2000.

[4] W. R. Mann, "Mean value methods in iteration," Proceedings of the American Mathematical Society, vol. 4, pp. 506-510, 1953.

[5] F. E. Browder and W. V. Petryshyn, "Construction of fixed points of nonlinear mappings in Hilbert space," Journal of Mathematical Analysis and Applications, vol. 20, pp. 197-228, 1967.

[6] Q. Liu, "Convergence theorems of the sequence of iterates for asymptotically demicontractive and hemicontractive mappings," Nonlinear Analysis, vol. 26, no. 11, pp. 1835-1842, 1996.

[7] K. Goebel and W. A. Kirk, "A fixed point theorem for asymptotically nonexpansive mappings," Proceedings of the American Mathematical Society, vol. 35, pp. 171-174, 1972.

[8] M. O. Osilike, A. Udomene, D. I. Igbokwe, and B. G. Akuchu, "Demiclosedness principle and convergence theorems for k-strictly asymptotically pseudocontractive maps," Journal of Mathematical Analysis and Applications, vol. 326, no. 2, pp. 1334-1345, 2007.

[9] T. H. Kim and H. K. Xu, "Strong convergence of modified Mann iterations," Nonlinear Analysis, vol. 61, no. 1-2, pp. 51-60, 2005.

[10] S. Reich, "Weak convergence theorems for nonexpansive mappings in Banach spaces," Journal of Mathematical Analysis and Applications, vol. 67, no. 2, pp. 274-276, 1979. 
[11] G. Marino and H.-K. Xu, "Convergence of generalized proximal point algorithms," Communications on Pure and Applied Analysis, vol. 3, no. 4, pp. 791-808, 2004.

[12] T. H. Kim and H. K. Xu, "Convergence of the modified Mann's iteration method for asymptotically strict pseudo-contractions," Nonlinear Analysis, vol. 68, no. 9, pp. 2828-2836, 2008.

[13] A. Genel and J. Lindenstrauss, "An example concerning fixed points," Israel Journal of Mathematics, vol. 22, no. 1, pp. 81-86, 1975.

[14] K. Nakajo and W. Takahashi, "Strong convergence theorems for nonexpansive mappings and nonexpansive semigroups," Journal of Mathematical Analysis and Applications, vol. 279, no. 2, pp. 372379, 2003.

[15] G. Marino and H.-K. Xu, "Weak and strong convergence theorems for strict pseudo-contractions in Hilbert spaces," Journal of Mathematical Analysis and Applications, vol. 329, no. 1, pp. 336-346, 2007.

[16] T. H. Kim, "Convergence of modified Mann's iteration methods for discrete asymptotically strict pseudo-contractive semigroups," in Proceedings of the Proceedings of the Asian Conference on Nonlinear Analysis and Optimization, pp. 139-162, 2008.

[17] W. Takahashi, Introduction to Nonlinear and Convex Analysis, Yokohama Publishers, Yokohama, Japan, 2009.

[18] J.-W. Peng and J.-C. Yao, "Two extragradient methods for generalized mixed equilibrium problems, nonexpansive mappings and monotone mappings," Computers \& Mathematics with Applications, vol. 58, no. 7, pp. 1287-1301, 2009. 


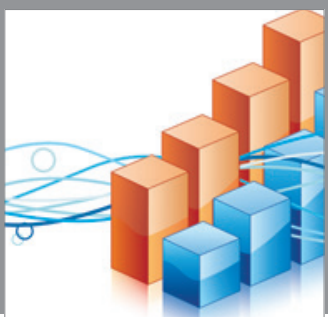

Advances in

Operations Research

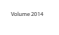

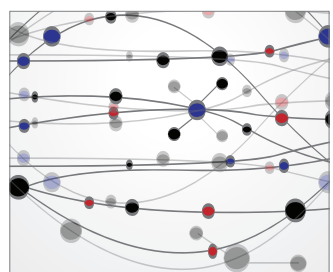

\section{The Scientific} World Journal
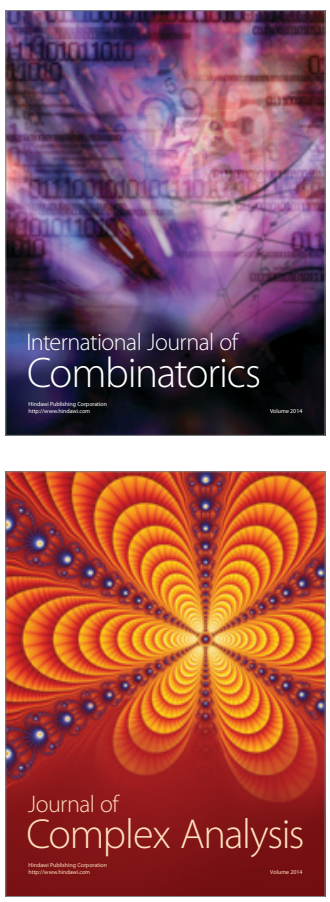

International Journal of

Mathematics and

Mathematical

Sciences
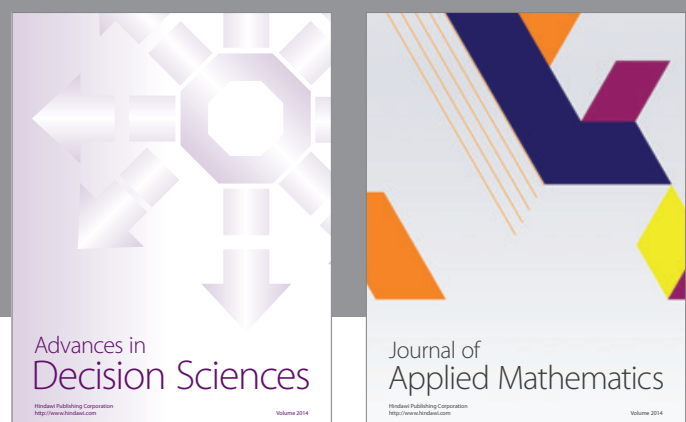

Journal of

Applied Mathematics
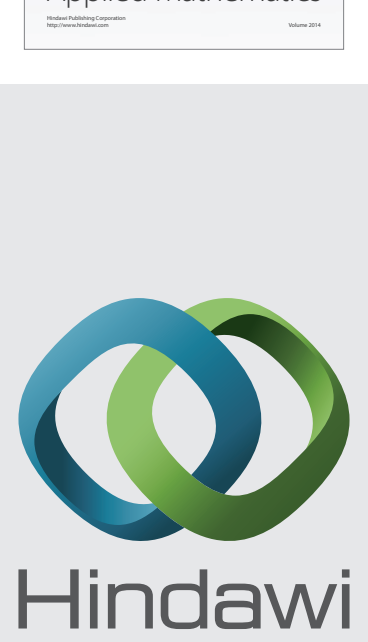

Submit your manuscripts at http://www.hindawi.com
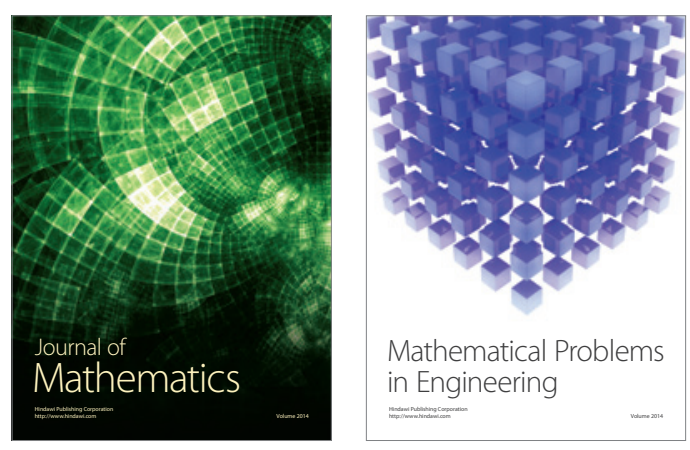

Mathematical Problems in Engineering
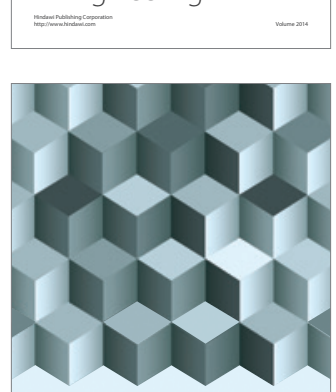

Journal of

Function Spaces
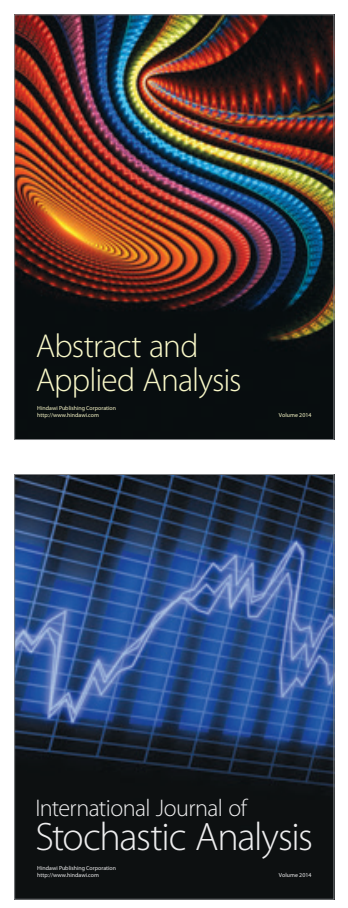

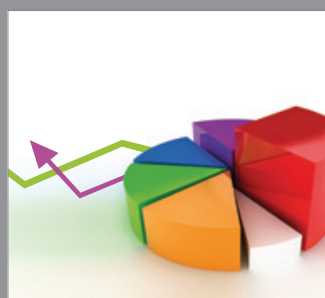

ournal of

Probability and Statistics

Promensencen
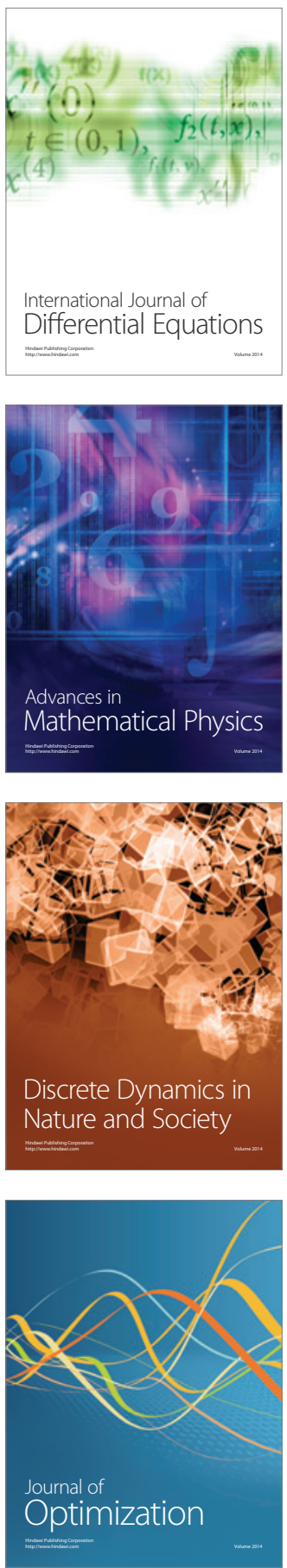